\title{
L'évolution de la dosimétrie opérationnelle à EDF*
}

\author{
R. DOLLO**
}

RÉSUMÉ Depuis plus de 10 ans, EDF a mis en œuvre une dosimétrie opérationnelle en utilisant des dosimètres électroniques à lecture directe. Cet exposé présente l'évolution du matériel et de l'utilisation des données informatisées à des fins de suivi des doses et de leur optimisation. Une réflexion globale est présentée sur les horizons futurs de la dosimétrie opérationnelle concernant les besoins d'EDF, l'application des futures directives européennes, et l'état d'avancement de projets industriels.

ABSTRACT Trends of operational dosimetry at EDF

For over 10 years, EDF has been conducting operational dosimetry by means of direct reading electronic dosimeters. The evolution of both the instrumentation and the utilization of computerized data for dose follow-up and optimization is presented. The overall prospects of operational dosimetry are considered in the light of EDF requirements, future european directives and industrial project developments.

\section{Introduction}

Au cours des 30 dernières années, la dosimétrie des rayonnements ionisants a évolué. Réservée à l'origine au radiodiagnostic et à la radiothérapie, elle s'est étendue à de nombreuses activités industrielles et, de ce fait, a dépassé le domaine médical. La multiplication des utilisateurs des rayonnements ionisants a entraîné la diversification des techniques de mesure de ces rayonnements. De même, l'objectif primordial de la surveillance individuelle a également évolué.

Au début, le but de la dosimétrie était de vérifier si les doses maximales admissibles (DMA) fixées par les normes de base n'étaient pas dépassées. Par exemple, il était recommandé de munir chaque travailleur d'un dosimètre à lecture directe si son travail l'exposait à un rayonnement dont le débit d'équivalent de dose dépassait largement $0,025 \mathrm{mSv} . \mathrm{h}^{-1}\left(2,5 \mathrm{mrem} \cdot \mathrm{h}^{-1}\right)$. Le dosimètre permettait d'évaluer les doses reçues, avec une incertitude de l'ordre de $50 \%$ et de s'assurer que la dose cumulée restait inférieure aux limites.

\footnotetext{
* Communication présentée aux journées SFRP "Les nouveaux enjeux de la radioprotection des travailleurs", Lyon 11-12 juin 1991.

** EDF, Service de la production thermique, Département sécurité-radioprotection-environnement (DSRE), BP 114, 93203 Saint-Denis Cedex 1.
} 
Le principe d'optimisation des doses recommandé par la publication CIPR 26 [3] a modifié le rôle de la dosimétrie. II ne s'agit plus de vérifier le respect des limites, il faut également que la dose reçue soit à un niveau aussi bas qu'il est raisonnablement possible (ALARA).

Depuis plus de 10 ans, EDF a mis en œuvre une dosimétrie opérationnelle. Cet exposé a pour but de montrer l'évolution des moyens techniques et de leurs utilisations, notamment grâce au développement des dispositifs électroniques et informatiques.

\section{Dosimétrie réglementaire - Dosimétrie opérationnelle}

Les textes réglementaires français [8-10] prennent en compte les principes fondamentaux émis par la Commission internationale de protection radiologique (CIPR) tels qu'ils sont précisés dans les directives européennes [4-6], à savoir :

- la limitation des expositions individuelles,

- l'optimisation de la radioprotection.

La dosimétrie opérationnelle existe depuis plusieurs années, en fait, depuis la mise en service des stylos électromètres à "lecture directe". Le concept de la dosimétrie opérationnelle a été introduit pour pallier l'impossibilité technique de la dosimétrie réglementaire par film photographique à accéder à la connaissance rapide de la dose. Pour répondre au principe de réduction des doses, les employeurs ont été amenés à utiliser des moyens de dosimétrie individuelle à réponse rapide et à mettre en œuvre des systèmes informatisés de plus en plus performants pour gérer les doses de rayonnements reçues par le personnel.

La dosimétrie opérationnelle a un double rôle :

- pour l'employeur et le travailleur, elle permet l'analyse périodique des postes de travail de façon à maintenir les expositions individuelles et collectives à un niveau aussi bas qu'il est raisonnablement possible ;

- pour le travailleur, elle lui permet de connaître en temps réel la dose intégrée et de réagir immédiatement pour faire cesser une exposition anormale.

Actuellement, le dosimètre opérationnel est à considérer comme un complément indispensable au dosimètre-film réglementaire. 


\section{3. Évolution de la dosimétrie opérationnelle}

Comme tout produit fabriqué, l'évolution de la dosimétrie opérationnelle est fonction de deux acteurs principaux :

- le constructeur qui vise un marché et souhaite améliorer sans cesse son produit pour le commercialiser plus facilement; dans le domaine de la dosimétrie, la concurrence est importante et les efforts des constructeurs conduisent à améliorer sans cesse les performances techniques ;

- le client qui définit son besoin en fonction du retour d'expérience du matériel utilisé et du rôle qu'il souhaite faire jouer à la dosimétrie opérationnelle ; son besoin est généralement défini dans un cahier des charges auquel devra satisfaire l'équipement de dosimétrie, le client définit des fonctions, il veut disposer d'un produit performant répondant à ses attentes et au moindre coût.

L'évolution de la dosimétrie opérationnelle est due également aux rencontres entre exploitants et constructeurs lors de réunions scientifiques, congrès ou sur le terrain.

\section{1 Évolution technique du dosimètre}

\subsubsection{Dosimétrie gamma}

L'ancêtre du dosimètre opérationnel s'appelle le stylo-dosimètre (stylo électromètre). C'est un condensateur qui doit être chargé avant utilisation (remise à zéro). Son état de décharge, représentatif de la dose reçue, est visualisé par un fil de quartz très fin, se déplaçant devant une graduation. Cet appareil simple, bon marché, a l'inconvénient d'avoir d'une précision de lecture et une gamme limitées. Pour couvrir une gamme plus large et signaler au porteur qu'il a séjourné dans un endroit à débit de dose élevé, celui-ci doit porter deux stylos dosimètres, avec une connaissance de la dose toujours a posteriori.

Les centrales nucléaires de la filière graphite-gaz sont encore équipées de ce type de dosimètre. En 1978, la centrale nucléaire de Fessenheim fut dotée de ce type de dosimètre. le procédé fut abandonné plus tard à cause des problèmes rencontrés lors de la saisie de la lecture optique du dosimètre. La centrale de Bugey fut la première à utiliser un dosimètre électronique de poche. La lecture de la dose est faite en actionnant un bouton poussoir. La mesure est affichée à quatre chiffres avec indication de dépassement de gamme (> 99,99 mSv). On associe au dosimètre tous les éléments nécessaires à la gestion automatique des doses : lecteur (lecture de la dose, remise à zéro), chargeur de batteries (contrôle du temps d'autonomie), unité centrale reliée aux lecteurs, imprimante.

Les difficultés de la lecture et le risque d'erreurs pour les faibles valeurs de dose ont amené les constructeurs à mettre au point des dosimètres électroniques donnant directement un affichage numérique de la 
dose permettant de concilier lecture directe, précision et large gamme de lecture. La mise en service dans les années 80 du palier CP1 s'est faite avec une dosimétrie électronique définie suivant un cahier des charges écrit par EDF.

La conception des dosimètres au cours des 10 ans écoulés a évolué selon les points principaux suivants :

Détection : l'évolution technologique de la fonction "détection" s'est déroulée en trois périodes:

1981 : détection par tube GM,

1985 : détecteur silicium et microprocesseur,

1989 : détecteur silicium et microprocesseur à haute intégration (circuit ASIC, hybridation).

Les premiers dosimètres utilisent des compteurs Geiger-Müller. Le passage au détecteur à semi-conducteur (diode silicium) sur le palier P'4 a permis d'améliorer la linéarité de la réponse en énergie et la précision des mesures dans les débits de dose élevés. Des dosimètres de ce type ont été développés pour une utilisation spécifique en situation accidentelle (linéarité jusqu'à $20 \mathrm{~Sv} / \mathrm{h}$ ) ; ils équipent tous les sites (40 dosimètres par site).

Caractéristiques mécaniques : $195 \mathrm{~g}$ (cahier des charges)... $120 \mathrm{~g}$ aujourd'hui. La miniaturisation du dosimètre est due à l'encombrement plus réduit du détecteur et à une consommation très faible qui permet d'atteindre aisément une autonomie d'un an avec pile autonome ou de réduire la capacité de la batterie.

Caractéristiques dosimétriques : Celles-ci ont été définies dans une norme CEI qui sera publiée prochainement [2] (Norme CEI 45B).

Caractéristiques opérationnelles : Ce caractère est essentiellement lié à la rapidité de l'information et à la gestion informatisée. Les constructeurs ont équipé leurs dosimètres des fonctions suivantes :

- mesure et affichage de l'équivalent de dose et du débit d'équivalent de dose,

- indication du débit d'équivalent de dose par éclat lumineux,

- alarmes sonores sur l'équivalent de dose et de débit de dose,

- gestion des seuils d'alarmes,

- capacité d'enregistrer l'évolution de la dose (historique).

Les dosimètres électroniques actuels possèdent suffisamment de fonctions à associer à un logiciel pour être capables de gérer immédiatement les doses reçues par le personnel.

\subsubsection{Dosimétrie neutron}

Actuellement, il n'existe pas de dosimètre individuel capable d'enregistrer les doses neutrons dans les conditions de travail de nos établissements. Aussi est-on dans l'obligation de les comptabiliser par le calcul du temps et du débit d'équivalent de dose neutron ambiant délivré par un radiamètre neutron. En effet, il existe bien des dosimètres individuels 
passifs (TLD notamment utilisés au Commissariat à l'énergie atomique (CEA) mais ils ne peuvent être que difficilement utilisés dans le bâtiment réacteur car le spectre neutron y est variable. Par contre, de tels dosimètres seront peut-être retenus comme dosimètres neutrons individuels lors des évacuations de combustibles (essais en cours). S'agissant de dosimètres passifs, la connaissance de la dose est acquise a posteriori (comme pour les stylos-dosimètres).

Bien que des recherches soient en cours, la réalisation d'un dosimètre individuel neutrons électronique ne semble pas devoir être effectuée dans un avenir proche : en effet, l'augmentation récente des facteurs de qualité accroît la difficulté de résoudre le problème posé par l'antagonisme sensibilité - miniaturisation.

\section{2 Évolution de l'application informatique}

\subsubsection{Historique}

Avant 1980, la gestion des doses se faisait manuellement. A partir de 1980, grâce à l'utilisation du dosimètre électronique, l'enregistrement des doses se fait automatiquement en sortie de zone contrôlée avec affectation manuelle d'un code d'activité pour relier la dose à une intervention en zone contrôlée. Dès 1985, la mise en place d'une gestion individualisée des doses et d'une gestion des accès en zone contrôlée est réalisée sur les nouveaux sites nucléaires. Toutes les restitutions permettent de réaliser le bilan des doses prises sur le site, mais l'exploitant ne peut détecter les anomalies que le lendemain après lecture des disquettes.

A partir de 1986, on peut suivre l'évolution de la dosimétrie par spécialités des agents EDF et d'entreprises extérieures en introduisant cinq codes d'activités professionnelles : mécanique-chaudronnerie, électricitéinstrumentation, contrôle-essais, servitudes, calorifuges.

Dès 1990, la direction du Service de la protection thermique (SPT) estime qu'il est de la responsabilité d'EDF de connaître la situation dosimétrique des agents d'entreprises extérieures. Une étude est lancée pour prévoir l'outil nécessaire au suivi des doses reçues par un même individu sur l'ensemble des sites EDF. (La dosimétrie des travailleurs extérieurs est reprise au paragraphe 3.2.3.)

\subsubsection{Dosimétrie opérationnelle en temps réel (fig. 1)}

Aujourd'hui, de nouveaux besoins liés à l'exploitation des équipements de dosimétrie opérationnelle apparaissent :

- le contrôle d'accès en zone contrôlée en temps réel suivant certains critères de radioprotection;

- la gestion en temps réel des doses reçues par les agents et par chantier.

Ainsi est née la dosimétrie opérationnelle en temps réel. 


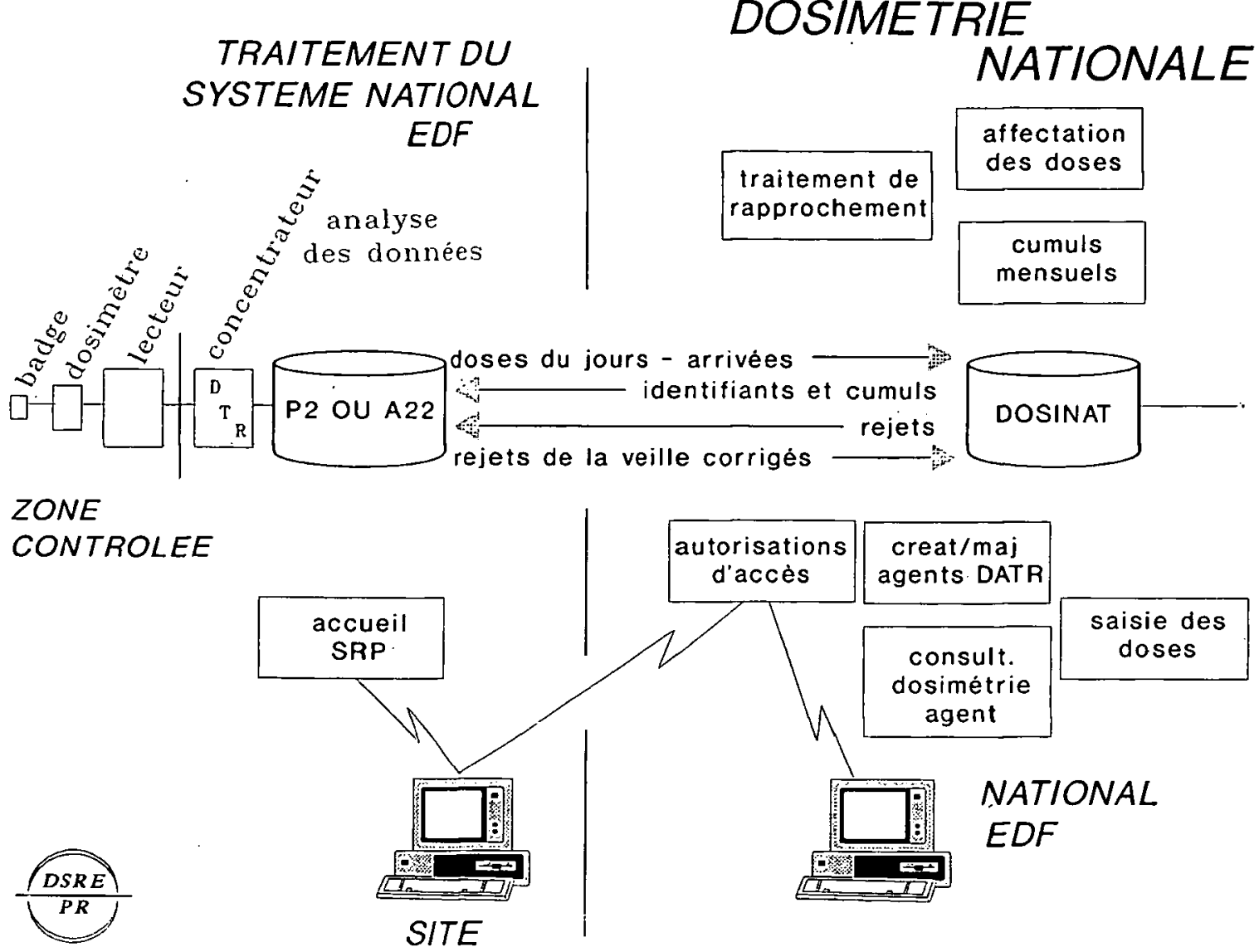

Fig. 1. - Dosimétrie opérationnelle

Les objectifs de la dosimétrie opérationnelle en temps réel sont les suivants :

a) Contrôle d'accès en zone contrôlée

L'objectif principal est l'automatisation du contrôle d'accès actuellement assuré par le gardien en entrée de zone contrôlée. Une personne peut se déplacer sur un site nucléaire grâce à son badge et aux informations qui y sont codées. Pour accéder aux zones contrôlées, il sera désormais nécessaire que la personne possède son badge, mais soit aussi autorisée par la SRP (section de radioprotection) qui codifiera dans un fichier informatique les autorisations nécessaires et les dates de validité de ces autorisations. A titre d'exemple, citons certains critères de radioprotection :

- habilitation PR1, PR2,

- date de validité (visite médicale, aptitude catégorie $A, B$ ),

- crédit de dose disponible,

- gestion des accès de certains chantiers (cas RGV Dampierre 1).

\section{b) Gestion des doses en temps réel}

Les doses sont cumulées sur diverses périodes paramétrables par l'opérateur. Au cours du travail, si un des crédits de dose rentré en mémoire dans le dosimètre vient à être atteint, l'agent est prévenu immédiatement par une alarme. 


\section{c) Gestion des codes travaux}

Pour mieux connaître les chantiers coûteux en doses et analyser les différentes phases du chantier afin d'appliquer une démarche d'optimisation, il est nécessaire de créer un découpage analytique des travaux réalisés en zone contrôlée. Ce découpage est réalisé à l'aide de codes travaux. La codification permet de contrôler le coût dosimétrique des travaux en fonction de différents critères, par exemple : zone géographique, chantier, spécialité des intervenants... Chaque code fait l'objet d'une autorisation d'utilisation pour éviter des anomalies résultant d'une mauvaise imputation de la part des intervenants. Le système fonctionnant en temps réel, toute anomalie est détectée instantanément.

La dosimétrie opérationnelle en temps réel est une nouvelle étape. Deux sites nucléaires (Bugey et St-Alban) et la base chaude opérationnelle des Tricastin (BCOT) sont équipés. On attend le retour d'expérience des systèmes existants pour envisager une généralisation à l'ensemble des sites.

\subsubsection{Dosimétrie des travailleurs extérieurs}

Les agents d'entreprise ont pour valeur reconnue de leur cumul en dose celle résultant du dernier développement de leur film dosimétrique reportée par le médecin du travail sur leur carnet DATR. La mise à jour du carnet par le médecin demande 2 à 3 mois et les valeurs de la dosimétrie opérationnelle indiquées par les agents ne sont pas toujours exactes. Aujourd'hui, bien que la responsabilité d'EDF ne soit pas engagée en cas de dépassement des normes, cette situation n'est pas satisfaisante.

La récente directive européenne [7] donne des responsabilité à l'exploitant comme à l'employeur en matière de suivi dosimétrique. Pour répondre à ce besoin, EDF a défini un système informatique de suivi national de la dosimétrie des agents intervenant en zone contrôlée (E.DF et entreprises) à partir des éléments fournis par la dosimétrie opérationnelle. Cette application nommée DOSINAT a pour objectifs majeurs :

- de mettre à la disposition des sites la connaissance du cumul des doses reçues par l'agent sur l'ensemble des sites EDF (passé dosimétrique) ;

- de constituer l'historique dosimétrique EDF sur 5 ans ;

- d'élaborer des statistiques opérationnelles (optimisation) ;

- de pouvoir être intégrée au futur système inter-entreprises (français et européen).

La mise en service de cette application est prévue au $1^{\text {er }}$ janvier 1992. Ce système national suivra seulement la dosimétrie opérationnelle et n'effectuera aucun rapprochement avec la dosimétrie film. L'architecture informatique est présentée figure 1. 


\section{Perspectives de la dosimétrie opérationnelle}

1) Les besoins à court terme d'EDF dans le domaine de la dosimétrie opérationnelle ont été définis dans le programme d'actions de réduction de doses présentées par $P$. Boussard [1]. La dosimétrie opérationnelle est appelée à jouer un rôle important dans la maîtrise des doses et l'amélioration des méthodes de travail en généralisant la mise en œuvre d'une dosimétrie par chantier. En effet, l'analyse fine des doses prises au cours des chantiers doit faciliter la recherche des écarts entre prévision et résultat pour porter les efforts sur des phases de chantiers bien ciblés.

Cette analyse est rendue possible grâce aux développements récents de la centralisation des données et de la possibilité d'un traitement en "temps réel". Moyennant quelques modifications au niveau des lecteurs d'entrée et de sortie de zone contrôlée, il sera possible de mettre en exploitation une dosimétrie en temps réel. Pour éviter les erreurs humaines lors de l'imputation des codes d'activité, une étude est en cours pour définir un moyen de lecture "à la volée" afin de saisir sur le lieu d'intervention réel les doses prises par le travailleur.

L'association de la dosimétrie en temps réel et de l'application informatique DOSINAT permet d'harmoniser l'ensemble de la chaîne "dosimétrie opérationnelle". Ces deux évolutions feront qu'EDF possédera un outil complet permettant d'une part de faire face à ses nouvelles responsabilités vis-à-vis des travailleurs extérieurs et d'autre part d'engager une véritable politique ALARA sur les sites.

2) A plus long terme, sachant que la conception du dosimètre retenu pour la centrale de Chooz B (en service en 1994/95) ne présente pas une très grande évolution par rapport à celui du premier palier (CP1), on peut imaginer que le dosimètre de l'an 2000 sera, lui, bien différent. En plus des caractéristiques actuelles, le futur dosimètre pourrait présenter des éléments nouveaux :

- miniaturisation (taille, poids,...) de façon à faciliter le port ;

- échanges entre les utilisateurs et le système de lecture des doses ;

- suivi en cumul des doses (fonction historique) ;

- prise en compte des rayonnements $B$ de faible énergie ;

- mesure en champs mixte $\gamma$ et neutrons (après étude économique) ;

- baisse du coût par rapport aux générations anciennes ;

- possibilité d'être reconnu comme dosimètre réglementaire pour remplacer le dosimètre-film qui présente les inconvénients d'une réponse en temps différé ( 2 mois), d'être imprécis et d'un coût d'exploitation non négligeable.

Enfin, pour répondre à la directive européenne "dosimétrie des travailleurs extérieurs" qui donne à l'exploitant des responsabilités en matière de suivi dosimétrique [7], un dosimètre personnel assurant la gestion des cumuls de doses des travailleurs se déplaçant de site en site (EDF ou non) serait le bienvenu (sorte de carte de crédit dosimétrique). 
3) Enfin, je souhaite remercier les constructeurs français qui, grâce à leur dynamisme et à leur savoir-faire, ont contribué efficacement à l'évolution de la dosimétrie individuelle. Actuellement, des projets sont lancés sur la nouvelle génération de dosimètres dont l'élément nouveau est la miniaturisation de façon à intégrer la fonction dosimètrie avec d'autres fonctions (montres, badges...). L'innovation est source de progrès mais, pour qu'un produit en remplace un autre, il est indispensable d'être conscient de la primauté du client, de l'amélioration permanente de la qualité et de la nécessité de la diminution des coûts.

\section{RÉFÉRENCES}

[1] BOUSSARD P. - Résultats dosimétriques du parc des réacteurs REP d'EDF. Radioprotection, 1992, 27 (1) 63-67.

[2] COMMISSION ELECTRONIQUE INTERNATIONALE (CEI). - Instrumentation pour la radioprotection. Moniteur individuel à lecture directe d'équivalent de dose et/ou de débit d'équivalent de dose pour les rayonnements $X$, gamma et bêta d'énergie élevée. (CEI-45B). Genève : CEI, 1990.

[3] COMMISSION INTERNATIONALE DE PROTECTION RADIOLOGIQUE (CIPR). Recommandations... (Publication CIPR 26). Oxford : Pergamon press, 1978.

[4] COMMUNAUTES EUROPEENNES. - Directive du Conseil du 3 septembre 1984 modifiant la directive 80/836/Euratom en ce qui concerne les normes de base relatives à la protection sanitaire de la population et des travailleurs contre les dangers résultant des rayonnements ionisants. JOCE, 1984, L265, 4-156.

[5] COMMUNAUTES EUROPEENNES. - Communication de la Commission au sujet de la mise en œuvre des directives du Conseil 80/836/Euratom du 15 juillet 1980 portant modification des directives fixant les normes de base relatives à la protection sanitaire de la population et des travailleurs contre les dangers résultants des rayonnements ionisants et 84/467/Euratom, du 3 septembre 1984, modifiant la directive 80/836/Euratom. JOCE, 1984, C347, 9-12.

[6] COMMUNAUTES EUROPEENNES. - Proposition de directive du Conseil concernant la protection opérationnelle des travailleurs exposés aux rayonnements ionisants au cours de leur intervention dans les installations utilisant ces rayonnements. JOCE, 1990, C284, 132-137 et C321, 2-7.

[7] COMMUNAUTES EUROPEENNES. - Directive du Conseil du 4 décembre 1990 concernant la protection opérationnelle des travailleurs extérieurs exposés à un risque de rayonnements ionisants au cours de leur intervention en zone contrôlée. JOCE, 1990, L349, 21-23.

[8] FRANCE. Premier ministre. - Décret $n^{\circ} 86-1103$ du 2 octobre 1986 relatif à la protection des travailleurs contre les dangers des rayonnements ionisants. JORF, 1986, 12 octobre, 12295-12335.

[9] FRANCE. Premier ministre. - Décret $n^{\circ} 88-6623$ du 6 mai 1988 modifiant le décret $n^{\circ} 75-306$ du 28 avril 1975 relatif à la protection des travailleurs contre les dangers des rayonnements ionisants dans les installations de base, et rectificatif. JORF, 1988,8 mai, $6718-6726$ et 30 juillet, p. 9796.

[10] FRANCE. Premier ministre. - Décret $n^{\circ}$ 91-963 du 19 septembre 1991 modifiant le décret $n^{\circ}$ 86-1103 du 2 octobre 1986 relatif à la protection des travailleurs contre les dangers des rayonnnements ionisants. JORF, 1991, 21 septembre, p. 12392. 